Guardando Oltralpe: la lunga marcia verso l'ouverture à gauche. Modello da imitare o incognita da evitare?

En regardant de l'autre côté des Alpes : la longue marche vers l'ouverture à gauche, un modèle à imiter ou une inconnue à éviter?

Looking to the Other Side of the Alps: The Long Walk to the "Opening to the Left", a Model to Imitate or to Avoid?

\title{
Michele Marchi
}

\section{OpenEdition}

\section{Journals}

Edizione digitale

URL: http://journals.openedition.org/cei/2863

DOI: 10.4000/cei.2863

ISSN: 2260-779X

Editore

UGA Éditions/Université Grenoble Alpes

\section{Edizione cartacea}

Data di pubblicazione: 20 avril 2016

Paginazione: 77-95

ISBN: 978-2-84310-323-0

ISSN: 1770-9571

\section{Notizia bibliografica digitale}

Michele Marchi, «Guardando Oltralpe: la lunga marcia verso l'ouverture à gauche. Modello da imitare o incognita da evitare?», Cahiers d'études italiennes [Online], 22 | 2016, online dal 01 janvier 2017 consultato il 27 mars 2021. URL: http://journals.openedition.org/cei/2863 ; DOI: https://doi.org/ 10.4000/cei.2863 


\title{
GUARDANDO OLTRALPE: \\ LA LUNGA MARCIA VERSO L'OUVERTURE À GAUCHE. MODELLO DA IMITARE O INCOGNITA DA EVITARE?
}

\author{
Michele Marchi \\ Università di Bologna
}

\section{Il quadro di insieme e le specificità di Francia e Italia}

Il passaggio tra la fine degli anni Cinquanta e l'avvio degli anni Sessanta costituisce, da un punto di vista sistemico, un tornante per l'evoluzione di quella sfera geopolitica che si può definire l'occidente euro-atlantico ${ }^{\mathrm{I}}$. Il processo di ricostruzione e consolidamento economico, che ha potuto sfruttare i finanziamenti del piano Marshall e l'impulso della Ceca (oltre che l'ombrello protettivo del Patto atlantico), giunge ad un punto di svolta con la firma dei Trattati di Roma del marzo $1957^{2}$. Completata la ricostruzione, le principali democrazie dell'Europa occidentale si apprestano ad avviare una fase di intensa crescita che avrà effetti di medio-lungo periodo sull'evoluzione dei propri sistemi economico-sociali, con importanti riflessi anche su quelli politici. Se ancora una volta da un punto di vista sistemico è possibile affermare che, a partire dai primi anni Sessanta, si consolida la cosiddetta 'democrazia del benessere', in base alla quale la possibilità di consumo per il cittadino-elettore diventa un elemento decisivo per la costruzione della legittimazione delle forze politiche che si contendono la guida del governo ${ }^{3}$, in realtà osservando le specificità nazionali si può notare una certa sfasatura tra i tempi dell'economia e della società e quelli della politica.

I. Cfr. G. Bernardini e M. Marchi (a cura di), A cinquant'anni dal primo centro-sinistra: un bilancio nel contesto internazionale, "Ricerche di Storia Politica», $\mathrm{n}^{\circ}$ 2, 2014.

2. Cfr. F. Romero, Storia della guerra fredda, Torino, Einaudi, 2009.

3. P. Pombeni, La ragione e la passione. Le forme della politica nell'Europa contemporanea, Bologna, Il Mulino, pp. 47I-544, e S. Cavazza e E. Scarpellini (a cura di), La rivoluzione dei consumi: società di massa e benessere in Europa 1945-2000, Bologna, Il Mulino, 2010. 
Sull'altra sponda dell'Atlantico la ripresa dei consumi di massa e il rilancio del modello americano sono in una prima fase gestiti dagli otto anni di presidenza repubblicana di Eisenhower, per poi vivere un momento di definitivo rilancio con l'arrivo alla Casa Bianca di J. F. Kennedy. Da un punto di vista simbolico, l'insediarsi del giovane e telegenico presidente costituisce, perlomeno a livello di percezione collettiva, il punto più alto del trionfo del modello euro-atlantico, nel quale si mescolano modernizzazione, consumi di massa e trionfo dei diritti civili. Complice anche la sua drammatica morte, il mito di Kennedy rappresenta, almeno per una parte di opinione pubblica, nonché dell'élite progressista europea, una sorta di 'nuova frontiera' del liberalismo in grado di conciliare più libertà ma allo stesso tempo maggiore redistribuzione ${ }^{4}$. Per certi versi l'evoluzione politica è simile nel contesto britannico. Con i conservatori guidati da Macmillan impegnati a gestire il post-ricostruzione e poi scavalcati elettoralmente dai laburisti di Wilson e dal suo insistere sui temi della modernizzazione, della pianificazione e della redistribuzione, una sorta di versione 'continentale' del liberalismo kennediano. L'uscita di scena di Adenauer in Germania Ovest e la sua sostituzione con l'artefice della cosiddetta 'economia sociale di mercato' costituiscono il primo passo, nel 1963, per l'avvio della grande coalizione di tre anni dopos.

Se si passa ai contesti francese ed italiano, innanzitutto si nota che i sistemi politico-istituzionali post-bellici sono accomunati dalla loro natura, perlomeno in questa congiuntura, in parte estranea all'evoluzione in atto nell'area euro-atlantica. L'Italia della Repubblica post-I946 e la Francia della IV Repubblica hanno un'evoluzione costituzionale e politica assimilabile. Non solo le due costituzioni sono piuttosto simili, ma la presenza di importanti soggetti partitici 'anti-sistema' costringe le principali forze di governo a coalizzarsi, ad inizio anni Cinquanta, per riuscire a garantire la governabilità del Paese ed impedire l'arrivo al potere di forze intenzionate a sovvertirne le fondamenta. Il sistema repubblicano italiano per contrastare il Pci utilizza la formula del 'centrismo' degasperiano così come la Francia della IV Repubblica, per rispondere agli attacchi di comunisti e gollisti, ricorre alla politica di 'terza forza'. Il fallimento del tentativo di introdurre un premio di maggioranza per la coalizione vincente e la successiva

4. Cfr. D. Ellwood, Una sfida per la modernità, Roma, Carocci, 2012, pp. 219 sgg., e M. Nolan, The Transatlantic Century I880-2010, Cambridge, Cambridge UP, 20I2. Cfr. anche V. de Grazia, L'Impero irresistibile, Torino, Einaudi, 2006.

5. Per uno sguardo complessivo D. Diner, Raccontare il Novecento: una storia politica, Milano, Garzanti, 200I. Una lettura di estremo interesse, anche se molto centrata solo sul caso francese, J.-F. Sirinelli, Les Vingt Décisives, Paris, Fayard, 2007. 
uscita di scena di De Gasperi, possono essere considerati, nel contesto italiano, i due passaggi decisivi per l'avvio del lungo 'viaggio' che condurrà un decennio dopo all'ingresso dei socialisti nell'area di governo. In questo percorso gestito in una prima fase da Amintore Fanfani e poi dalla coppia Fanfani-Moro un momento senza dubbio chiave è rappresentato dall'effetto degli eventi di Budapest del 1956 e dalla successiva e definitiva presa di distanza del Psi di Pietro Nenni dall'alleanza con il Pci nel cosiddetto 'fronte popolare'. Ma il 1956 è un anno chiave anche per l'evoluzione politico-istituzionale francese. La guerra d'Algeria ha fatto irruzione con veemenza il ${ }^{\circ}$ novembre 1954 (il giorno della cosiddetta Toussaint rouge), ma è in seguito alla violenta contestazione al Primo ministro Mollet in visita ad Algeri il 2 febbraio 1956 che la Francia entra completamente nel conflitto, portando gli effettivi del suo esercito dispiegato in Algeria ad oltre 400 mila uomini. Da questo momento in poi la 'questione algerina' sarà centrale per l'evoluzione del sistema politico-istituzionale perlomeno sino a fine $1962^{6}$.

Dunque per certi aspetti due sistemi con le medesime problematiche a livello di governabilità e assenza di una reale forma di alternanza, si trovano a fare i conti con le nuove incognite del post-ricostruzione e a vivere nello stesso periodo 1958-1962 (1963 in Italia se si considera il centrosinistra organico e non quello con il sostegno in Parlamento dei socialisti nenniani, ma senza la presenza di ministri socialisti del febbraio 1962) una decisiva svolta. Insomma Parigi e Roma affrontano l'ingresso nella 'società del benessere' sfidate da una situazione di crisi strutturale o egemonica ${ }^{7}$.

Nel contesto francese, tra il 1958 e il 1962 si è di fronte ad una triplice svolta. Innanzitutto istituzionale, con il varo della nuova Costituzione a seguito del ritorno alla guida del Paese da parte di Charles de Gaulle, ultimo presidente del Consiglio della IV Repubblica (dopo esserne stato il primo), investito dei poteri costituzionali e dei pieni poteri in Algeria praticamente da tutte le forze politiche, eccetto il Pcf e alcune personalità di primo piano, tra le altre Pierre Mendès France e François Mitterrand ${ }^{8}$. Dopo il successo referendario del settembre 1958 , le elezioni del novembre 1958 non costituiscono una cesura sostanziale nel quadro partitico ${ }^{9}$. La seconda svolta

6. Cfr. M. Vaïsse (a cura di), De Gaulle et l'Algérie, I943-1969, Paris, Armand Colin, 2012.

7. Su questo concetto vedi F. Cammarano, Crisi politica e politica della crisi, in P. Pombeni (a cura di), Crisi, legittimazione, consenso, Bologna, Il Mulino, 2003, pp. 8I sgg.

8. Vedi S. Berstein e M. Winock (a cura di), La République recommencée, Paris, Seuil, 2008, pp. 34I-388.

9. Cfr. B. Lachaise, G. Le Béguec e F. Turpin (a cura di), Les élections législatives de novembre 1958 : une rupture?, Bordeaux, Presses universitaires de Bordeaux, $201 \mathrm{~s}$. 
consiste nel completamento del processo di decolonizzazione e nello specifico nella fine della guerra d'Algeria, con il cessate il fuoco, gli accordi di Evian poi ratificati da un successivo referendum. Infine la terza e ultima svolta è quella che si concretizza nel periodo marzo-novembre 1962, che vede l'avvicendamento tra Debré e Pompidou a Matignon, l'annuncio da parte di de Gaulle della volontà di procedere al 'completamento' della svolta istituzionale introducendo l'elezione diretta del presidente della Repubblica, non utilizzando i meccanismi di revisione costituzionale ordinari, ma sottoponendo la proposta a voto referendario. Il braccio di ferro tra tutti i partiti politici (eccetto il partito gollista) e il presidente in carica vede il successo di quest'ultimo sia al referendum di fine ottobre, sia alle legislative di novembre 1962 che segnano, questa volta a differenza del I958, una cesura per il sistema politico e partitico, che progressivamente si adatterà al nuovo modello istituzionale oramai definitivamente centrato sul primato dell'esecutivo rispetto al legislativo ${ }^{\mathrm{IO}}$.

L'evoluzione politico-istituzionale francese è osservata dai contemporanei italiani, si tratti dei principali commentatori, dei più noti costituzionalisti e dei principali protagonisti politici, con un sentimento di stupore, misto a timore. Su un punto tutti sembrano convenire: l'evoluzione centrata sul primato dell'esecutivo e della democrazia plebiscitaria non sono esportabili nel contesto 'speciale' dell'Italia ${ }^{\text {II }}$. Soltanto sull'onda della crisi del sistema politico italiano, a partire dai primi anni Ottanta, l'evoluzione 'gollista' delle istituzioni francesi comincerà ad interessare porzioni sempre più vaste della politica e dell'accademia italiana, sino ad arrivare ad una vera e propria 'moda del semipresidenzialismo' nella fase di più acuta crisi del sistema politico-istituzionale repubblicano sull'onda delle inchieste giudiziarie di Mani Pulite e del progressivo dissolversi dei partiti politici fondatori del sistema repubblicano, anche successivo all'esaurirsi della guerra fredda ${ }^{\mathrm{I2}}$.

Io. G. Richard e J. Sainclivier (a cura di), Les partis et la République : la recomposition du système partisan (1956-1976), Rennes, PUR, 2008; J. Garrigues, S. Guillaume e J.-F. Sirinelli (a cura di), Comprendre la Ve République, Paris, PUF, 2010 e in generale J. Vigreux, Croissance et contestations 1958-198I, Paris, Seuil, 2014.

II. Cfr. A. Argenio, Da maggio a maggio. La Dc e l'avvento al potere di de Gaulle nel 1958 , "Italia contemporanea", $\mathrm{n}^{\circ}$ 270, 20I3, pp. II-32 e P. Milza (a cura di), De Gaulle et l'Italie, Rome, École française de Rome, 1997.

I2. R. Chiarini, La fortuna del gollismo in Italia. L'attacco della destra alla 'Repubblica dei partiti", "Storia Contemporanea", giugno I992; R. Chiarini, La fortuna del gollismo in Italia. Le suggestioni di una 'Seconda Repubblica', "Storia Contemporanea», aprile 1994 e G. Quagliariello, Gaullisme. Une classification impossibile, Paris, L'Harmattan, 2009. Di taglio più giuridico F. Lanchester, La Quinta Repubblica nel dibattito e nella prassi in Italia, Napoli, Jovene, 2009. Di taglio più politologico G. Pasquino e S. Ventura (a cura di), Una splendida cinquantenne: la Quinta Repubblica francese, Bologna, Il Mulino, 2010. 
Se dunque questa è in breve l'evoluzione istituzionale e politica nel contesto francese e se tali cambiamenti sono stati osservati con estrema attenzione nel contesto italiano, giova soffermarsi sulla congiuntura 1958I962, altrettanto importante nell'evoluzione del complesso sistema politico-istituzionale italiano.

Qui la svolta, almeno formalmente, non è istituzionale, ma è essenzialmente politica ed avviata da Amintore Fanfani, segretario del partito una volta uscito di scena De Gasperi e poi anche presidente del Consiglio dopo le elezioni legislative del maggio 1958. Ben presto il progetto fanfaniano di arrivare all'apertura a sinistra, cioè al coinvolgimento dei socialisti nell'area di governo, sfruttando le leve del governo (e del suo programma di riforme e di coinvolgimento dello Stato nell'economia) a scapito di quelle del partito (da lui accentrato e ridotto nella sua logica correntizia) naufragano proprio per una rivolta interna al suo stesso partito. Le sue doppie dimissioni (da presidente del Consiglio e da segretario del partito) del gennaio 1959, finiscono per ribadire la centralità della stessa Dc nelle dinamiche sistemiche italiane. Il consiglio nazionale del I4-I8 marzo 1959 convocato a Roma, presso la Domus Mariae, sancisce da un lato la nascita della nuova corrente di maggioranza della Dc, quella dei dorotei (in larga parte fanfaniani insoddisfatti della gestione del partito di quello che era il 'loro' segretario) e dall'altro l'avvio della lunga segreteria democristiana di Aldo Moro (ratificata poi dal Congresso di Firenze dell'ottobre successivo) ${ }^{\mathrm{r}}$. Da questo momento in poi è di nuovo un 'uomo di partito', come Aldo Moro, a gestire il lento e progressivo movimento di apertura a sinistra, che deve tenere conto certamente delle dinamiche interne al Partito socialista, profondamente diviso su questo punto, ma che deve allo stesso modo fare estrema attenzione alle complicate caratteristiche costitutive del partito di maggioranza relativa, che rappresenta l'asse portante del bipartitismo imperfetto (e bloccato) italiano ${ }^{\mathrm{I4}}$. La Dc è partito che federa sensibilità politiche, economiche e sociali profondamente differenti in nome di un'unità politica dei cattolici, della quale garanti ultimi sono le gerarchie ecclesiastiche. Ecco perché il costante lavoro di Moro dal marzo I959 sino al dicembre di cinque anni dopo è svolto lungo due direttrici. Da un lato garantire l'unità del partito unico dei cattolici. Dall'altro farsi garante, attraverso un'accorta e costante opera di 'informazione' diretta al pontefice

13. Vedi M. Marchi, Aldo Moro segretario della Democrazia cristiana. Una leadership politica in azione 19591964, "Mondo Contemporaneo", n 2, 2010, pp. I05-I36.

I4. Cfr. il classico G. Galli, Il bipartitismo imperfetto: comunisti e democristiani in Italia, Bologna, Il Mulino, 1966. 
Giovanni XXIII e ai principali responsabili vaticani per le questioni italiane (la cosiddetta 'politica ecclesiastica' morotea) del processo di apertura dell'area di governo ai socialisti. Nella convinzione di Moro questo processo dovrebbe permettere prima di tutto il consolidamento di un sistema democratico comunque menomato dall'impossibilità di giungere ad una fisiologica alternanza (a causa della 'scelta orientale' del Pci). In secondo luogo dovrebbe garantire il dispiegarsi di quelle necessarie 'riforme' economico-sociali nella direzione di una maggiore redistribuzione e di una più attenta trasformazione della società italiana da una mera dimensione di sussistenza ad una più completa e articolata dimensione di democrazia del benessere e dei consumi, seppur temperati dagli insegnamenti della dottrina sociale della Chiesa ${ }^{15}$.

Obiettivo del presente contributo è offrire un primo punto di vista su come l'opinione pubblica francese in generale ${ }^{16}$ e più nello specifico quella delle pubblicazioni legate ai principali partiti politici ${ }^{17}$, hanno osservato e commentato nel periodo 1962-1963 la fase conclusiva del tentativo di apertura a sinistra, da intendersi sia come via originale per passare dalla fase della ricostruzione a quella della democrazia del benessere, sia come nuova alternativa all'entrata in crisi del centrismo degasperiano e dunque giungere ad una nuova declinazione per il sistema bloccato ${ }^{18}$.

Per cercare di circoscrivere un'analisi altrimenti eccessivamente vasta e dispersiva si sono scelti quattro momenti chiave dell'operazione di apertura a sinistra. Il primo riguarda il congresso democristiano di Napoli del I962 e la successiva nascita del IV governo Fanfani appoggiato dalla maggioranza dei deputati socialisti. In secondo luogo è analizzata l'elezione di Segni alla presidenza della Repubblica. Sono poi osservate da vicino le

I5. Vedi M. Marchi, Moro, la Chiesa e l'apertura a sinistra. La 'politica ecclesiastica' di un leader 'post-dossettiano', "Ricerche di Storia politica», $\mathrm{n}^{\circ}$ 2, 2006, pp. I47-I79 e Id., La Dc, la Chiesa e il centro-sinistra. Fanfani, Moro e l'asse vaticano' 1959-I962, "Mondo contemporaneo", $\mathrm{n}^{\circ}$ 2, 2008, pp. 4I-90. Vedi anche P. Pombeni, I partiti e la politica dal $\mathrm{I}_{94} 8$ al 1963 , in G. Sabbatucci e V. Vidotto (a cura di), Storia d'Italia, vol. V, Roma-Bari, Laterza, 1997, pp. I27-25I; P. Scoppola, La Repubblica dei partiti, Bologna, Il Mulino, I99I, pp. 233 sgg., e in generale tutta la prima parte di P. Craveri, La Repubblica dal 1958 al 1992, Torino, Utet, 1995.

I6. "Le Monde» è stato utilizzato come base della riflessione così come sono presentati anche alcuni contributi da «L'Express». Un interessante sguardo dalla Francia sull'evoluzione storico-politica, ma anche economica e sociale, dell'Italia nel post 1945 è stata offerta alcuni anni fa dal volume a cura di Marc Lazar, L'Italie contemporaine de 1945 à nos jours, Paris, Fayard, 2009.

17. Sono stati presi in esame "La Croix» come giornale rappresentativo delle sensibilità più legate istituzionalmente alla Chiesa di Francia, «Forces Nouvelles», periodico ufficiale del Mrp, «La Nation» per l'Unr, «Le Populaire» per la Sfio e «L'Humanité» come rappresentativo del mondo comunista. L'evoluzione del sistema politico-istituzionale già richiamata vede in grave crisi in particolare Mrp e Sfio, cardini della IV Repubblica e questo si riflette anche nelle loro pubblicazioni. Non a caso non esiste un quotidiano per il Mrp e «Le Populaire» cessa le sue pubblicazioni nel giugno 1963, come corollario alla profonda crisi della Sfio.

I8. M. L. Salvadori, Storia d'Italia. Crisi di regime e crisi di sistema, I86I-2013, Bologna, Il Mulino, 2013. 
elezioni legislative dell'aprile del 1963. E infine è naturalmente analizzata la fase conclusiva, con le reazioni al varo del primo governo 'organico' di centro-sinistra del dicembre 1963.

\section{Da Napoli, finalmente la svolta?}

Nel complesso l'opinione pubblica francese si attende dal nuovo anno, 1962, e nello specifico dal Congresso democristiano di fine gennaio, una svolta sulla nuova formula di governo. Non a caso "Le Monde», in un fondo del 9 gennaio 1962, non esita ad affermare: "Beaucoup de conditions paraissent réunies pour que l'année qui s'ouvre soit marquée par un profond bouleversement de la vie politique italienne. La question qui se pose est toujours la même : c'est celle de l'ouverture à gauche» ${ }^{19}$. Correttamente «Le Monde» si sofferma a commentare che, dopo le affermazioni del segretario Aldo Moro il 22 novembre precedente a Tribuna politica (egli ha parlato di un accordo con il Psi di Nenni esclusivamente su un programma concordato), il vero passaggio decisivo è tutto interno alla Dc. E ci si chiede, concludendo il commento, se la componente di 'destra' (Scelba, Gonella, Pella, Andreotti) accetterà questa declinazione e lascerà la coppia Fanfani-Moro libera di gestire l'accordo 'programmatico' con i socialisti nenniani. I toni poi di «Le Monde» diventano quasi entusiastici nel commentare la chiusura del Congresso Dc a Napoli. Per il quotidiano parigino il Congresso democristiano ha avuto un chiaro vincitore: Amintore Fanfani e con lui l'avvio di una nuova fase politica nel Paese ${ }^{20}$.

M. Fanfani a remporté mercredi, dernier jour des débats du huitième congrès de la Démocratie chrétienne, un succès spectaculaire. À la fin du discours quill a prononcé, [...] les délégués lui ont fait une longue ovation. La cause est donc entendue : la Démocratie chrétienne, après avoir louvoyé pendant trois lustres entre les partis centristes, abandonne les libéraux à leur sort, et se propose de contracter une alliance avec les socialistes nenniens. Ce projet de renversement de la majorité répond aux exigences des saragatiens et des républicains - et correspond aux propositions avancées par M. Nenni ${ }^{21}$.

19. Les démocrates chrétiens italiens et l'ouverture à gauche, "Le Monde», o9/or/1962.

20. Da notare che qualche giorno prima "L'Express» ha dedicato un lungo contributo alla 'svolta' italiana, centrando però tutta l'attenzione sulla figura di Nenni e sul ruolo del Psi nell'apertura a sinistra. «Le parti socialiste italien va tenter une aventure politique exceptionnelle, en accordant son soutien à son vieil adversaire, le parti démocrate-chrétien, au pouvoir depuis I6 ans» (M. Bosquet, Le grand pari, «L'Express», oI/O2/1962).

2I. J. D'Hospital, M. Fanfani chef de file des partisans de l'ouverture à gauche remporte un net succès, "Le Monde", 02/02/I962. 
Sempre seguendo questa linea, la nascita del IV governo Fanfani, con l'appoggio parlamentare dei socialisti nenniani (ma non la partecipazione di ministri socialisti), è descritto come un «mythe qui est en train de devenir réalité», come un evento che potrà "changer beaucoup de choses en Italie»" 22.

Se dal principale organo di stampa francese passiamo alle principali pubblicazioni di partito, il quadro si arricchisce di sfumature. Su un punto gli osservatori dei principali partiti politici sembrano essere concordi: il Congresso Dc di Napoli costituisce un momento di svolta, un punto di non ritorno non soltanto per la Democrazia cristiana ma per l'intero sistema politico italiano. L'idea dell'apertura a sinistra a questo punto è 'irreversibile'. Stabilita la certezza della formula, è necessario riempirla di significato effettivo. Su tale questione i giudizi divergono in maniera anche piuttosto rilevante. Che cosa significa insomma 'centro-sinistra', nel concreto e al di là della formula Dc più Psi, più partiti laici minori senza i liberali?

Se si comincia ad osservare il quadro da sinistra si nota la scarsa attenzione mostrata dall'organo di riferimento della Sfio, «Le Populaire», che si limita a descrivere l'esito del Congresso di Napoli del gennaio 1962 e la successiva formazione del quarto governo Fanfani (sostenuto da socialdemocratici e repubblicani e con l'astensione dei socialisti) come la vittoria di Fanfani e del suo progetto avviato sin dal suo arrivo alla guida del partito nel 1954 come successore di De Gasperi ${ }^{23}$.

Ben più articolata è l'analisi offerta da «L'Humanité». L'organo ufficiale del Pcf definisce quella operata da Moro al Congresso di Napoli una vera e propria 'discontinuità. Si è di fronte dunque ad un tornante sistemico in larga parte frutto della reazione democristiana alla costante crescita del movimento operaio guidato dal Pci in Italia. Al momento della formazione del nuovo governo Fanfani l'orizzonte è aperto a differenti ipotesi. Soprattutto "L'Humanité» individua due passaggi chiave nell'immeditato futuro. Punto primo sarà decisivo valutare quali e quante concessioni i democristiani saranno disposti a fare per ottenere l'ingresso effettivo dei nenniani nell'area di governo. In secondo luogo sarà importante vedere fino a che punto gli stessi socialisti saranno disposti a difendere una serie di principi «socialisti», giudicati inderogabili. Il ruolo del Pci diventa a questo punto determinante. I comunisti italiani dovranno operare affinché la Dc conceda il più possibile se vuole davvero avere l'appoggio dei socialisti. D'altra parte sempre il maggiore partito della sinistra dovrà impe-

22. L'ouverture à gauche est faite en Italie, «Le Monde», 23/02/1962.

23. Ouverture à gauche en Italie, "Le Populaire», 22/02/1962. 
gnarsi affinché l'operazione voluta dalla coppia Fanfani-Moro perda gran parte del suo appeal e soprattutto non venga presentata come un'apertura a sinistra', dal momento che:

[...] un véritable tournant à gauche implique une participation directe et effective des travailleurs au pouvoir. Si les démocrates chrétiens et une partie des socialistes pensent pouvoir s'engager sur une voie différente, ils auront à affronter l'action décidée et organisée de la classe ouvrière et de l'ensemble des travailleurs' ${ }^{24}$.

L'idea del necessario contrasto alla crescita del Pci da condurre sui temi, sulle questioni e le politiche concrete, è quella che muove il commento di «La Croix» al Congresso Dc di Napoli. Per dissolvere il blocco social-comunista e contrastarne l'avanzata, l'apertura a sinistra può essere un effettivo antidoto. Tale 'necessità' del centro-sinistra non contribuisce però a diminuirne le difficoltà di realizzazione. Si sottolineano le difficoltà che Fanfani dovrà affrontare soprattutto all'interno del suo stesso partito, con l'ala 'destra' totalmente contraria e quella 'sinistra' che considera quella dell'apertura a sinistra come l'irripetibile occasione per introdurre nel Paese forti dosi di riformismo. Ma soprattutto si avanzano non pochi dubbi sul ruolo del socialismo nenniano. E ci si chiede esplicitamente quali garanzie potrà offrire su temi cruciali in particolare relativi alla politica estera. Rispetto alle linee di politica internazionale del Psi, ci si chiede, infatti, se il socialismo nenniano possa realmente condividere con la Dc, una volta in un'alleanza organica di governo, i sentimenti di europeismo e atlantismo, così decisivi per definire la collocazione internazionale del Paese ${ }^{25}$.

La dimensione 'europea' o per meglio dire 'internazionale' dell'apertura a sinistra interessa particolarmente a "Forces Nouvelles»" ${ }^{26}$. Sia il Congresso Dc del gennaio 1962, sia la successiva formazione del governo Fanfani, narrano di un Paese intento a trovare la giusta formula per ottenere il "progresso senza rivoluzione». L'ingresso (seppur ancora solo ipotetico) dei socialisti nell'area di governo deve tramutarsi in un «evento» di portata europea, non perdendo però di vista le molte incognite che gravano su

24. Fanfani va essayer de constituer un gouvernement appuyé par les socialistes, «L'Humanité», 03/02/I962.

25. G. Huber, M. Fanfani va s'efforcer de constituer une nouvelle majorité, «La Croix», 03/02/1962.

26. Questo è in larga parte un riflesso sia della tradizionale vocazione europeista del Mrp, sia dell'evoluzione che il partito sta avendo a partire dalla fine degli anni Cinquanta. Sempre più in crisi come partito di massa all'interno dei confini nazionali, il Mrp cerca di connotarsi come 'partito dell'Europa'. La dimensione tenderà ad accentuarsi dopo la seconda traumatica rottura con il generale de Gaulle, perpetrata proprio a partire dal disaccordo sulla condotta dello stesso presidente sui temi della politica europea. Su tutto questo, v. S. Berstein, J.-M. Mayeur e P. Milza (a cura di), Le MRP et la construction européenne, Bruxelles, Éditions Complexe, I993; M. Marchi, Les centristes français et la construction européenne du MRP à Valéry Giscard d'Estaing, in Consensus and European Integration. An Historical Perspective, Bern, Peter Lang, 2012, pp. 8I-96. 
di esso. Ecco allora che servono «prudenza» e «audacia» per condurre in porto l'operazione, facendo molta attenzione ad evitare che possa consolidarsi, all'interno della nuova alleanza, lo spirito anti-europeo del socialismo italiano ${ }^{27}$. È in particolare il padre nobile della «democrazia cristiana alla francese» a suonare il campanello d'allarme su questo punto. Étienne Borne, insistendo proprio sul significato e la dimensione «internazionale» dell'operazione, non esita però ad affermare che:

M. Nenni n'est pas un Spaak ou un Guy Mollet, et sa ferveur européenne peut faire question. Là est le véritable danger d'une ouverture à gauche qui ferait des concessions moins au socialisme proprement dit qu'à un néo-capitalisme neutraliste, anti atlantique et qui ne serait que verbalement européen. S'ouvrir à une fausse gauche, on sait que le péril existe et qu'il porte même un nom propre, ce serait manquer l'ouverture à gauche ${ }^{28}$.

Inoltre «Forces Nouvelles» utilizza il caso italiano dell'apertura a sinistra per riflettere sulle grandi difficoltà che sta vivendo il Mrp, partito della IV Repubblica impegnato in un complicatissimo tentativo di adattamento alle nuove istituzioni volute da de Gaulle. Quello italiano diventa una sorta di 'modello', in cui opera un partito rispetto al quale il Mrp, al solito, guarda con un misto di rispetto e invidia reverenziale.

Heureuse Italie, qui peut nous exposer en toute liberté la crise de conscience d'un grand parti, capable de faire à grande échelle son "autocritique" sans destitution ni mort d'homme, avec la seule volonté de tracer une ligne qui soit un progrès sans révolution. Par un juste retour des choses, voici que l'Italie démocratique montre à la France un chemin, une sauvegarde possible. Elle lui rappelle que les hommes ont moins d'importance que l'évolution du courant d'idées et que, dans l'état actuel des choses, un regroupement de centre-gauche peut seul s'opposer avec efficacité à tous les fascismes et faire triompher la liberté29.

Dunque nel complesso il Congresso democristiano del 1962 e la successiva complicata formazione del quarto governo Fanfani costituiscono un'importante fase di mutamenti del sistema politico-istituzionale italiano, ricco di opportunità così come di incognite, destinate in parte a dissiparsi, come afferma "La Croix», in occasione del voto legislativo della primavera dell'anno successivo. Il quotidiano degli Assunzionisti non ha alcun dubbio: «La vera apertura a sinistra, se andrà in porto, sarà quella successiva al voto del $1963^{3{ }^{30}}$. Intanto però l'elezione del nuovo presidente della Repubblica, a seguito dello scadere del mandato di Gronchi, può ulteriormente complicare il quadro.

27. J.-P. Cornet, Les démocrates-chrétiens face à l'ouverture à gauche, «Forces Nouvelles», 25/01/1962.

28. E. Borne, L'ouverture à gauche, "Forces Nouvelles», 25/0I/1962.

29. J.-P. Cornet, Les démocrates-chrétiens face à l'ouverture à gauche, cit.

30. Fanfani : une majorité confortable, mais fragile, "La Croix", 13/03/1962. 


\section{La complicata elezione di Antonio Segni}

Come gestire la successione di Giovanni Gronchi? Un ruolo di primo piano spetta naturalmente a Moro, leader del partito di maggioranza relativa della coalizione di governo. Poiché nel corso del settennato gronchiano la presidenza della Repubblica era stata concepita ed identificata come strumento di destabilizzazione del sistema, Moro è convinto che serva un segnale di inversione di rotta. Decide così di 'consegnare alla destra', che peraltro egli aveva contribuito a non sovra-rappresentare nel quarto governo Fanfani, l'elezione del presidente della Repubblica. Fa così emergere la candidatura di Antonio Segni, il quale è eletto dopo una lunga battaglia parlamentare in cui i 'laici', con la candidatura di Giuseppe Saragat, oppongono la logica del bilanciamento delle 'aree politiche' (un cattolico alla guida del governo e un laico al Quirinale), a quella logica del 'bilanciamento delle ideologie' (un progressista al governo e un conservatore alla presidenza della Repubblica) a cui punta Moro ${ }^{31}$.

Secondo «Le Monde» l'elezione di Segni alla presidenza della Repubblica senza dubbio complica la futura nascita di un esecutivo con ministri socialisti. Ma ancor di più chiarifica, se ve ne era necessità, quanto l'evoluzione del sistema politico italiano non possa prescindere dall'evoluzione interna di quel movimento (e non partito, afferma «Le Monde») che è la Dc.

On n'avait jamais vu aussi clairement étalée cette vérité que la ligne de partage de la vie politique italienne passe au milieu du grand parti majoritaire. Combien de temps cette situation incommode pour la Démocratie chrétienne pourra-t-elle se prolonger ${ }^{32}$ ?

A proposito dell'elezione di Segni al sesto scrutinio il 6 maggio I962 su un punto sembrano convergere le analisi provenienti dai principali organi 'politici', anche se schierati su posizioni di solito diametralmente opposte. «La Croix» e «Forces Nouvelles» da un lato, sia pure con sfumature differenti, e dall'altro lato "L'Humanité» concordano su questo punto: l'elezione di Segni alla presidenza della Repubblica ha finalmente offerto una chiara immagine di cosa sia realmente la formula di centro-sinistra, insomma si è di fronte ad un momento chiarificatore.

Per «L'Humanité» dalla «scandalosa» elezione di Segni è emerso l'unico obiettivo della Democrazia cristiana nel momento in cui vuole costruire un'alleanza di centro-sinistra e cioè mantenere la maggiore forza popolare

3I. Cfr. P. Pombeni, I partiti e la politica dal 1948 al I963, cit. pp. 232-233; nello specifico sul passaggio dalla presidenza Gronchi a quella Segni, v. G. Mammarella e P. Cacace, Il Quirinale. Storia politica e istituzionale da De Nicola a Napolitano, Roma-Bari, Laterza, 20II, pp. 90 sgg.

32. Une situation incommode pour la Démocratie chrétienne, «Le Monde», 08/05/1962. 
del Paese fuori dal governo dello stesso. Il carattere solamente «strumentale» dell'alleanza di centro-sinistra, secondo "L'Humanité», è stato reso evidente dalla scelta della coppia Fanfani-Moro di non appoggiare il candidato sostenuto dalle forze di sinistra (compreso il Pci), cioè Giuseppe Saragat, peraltro leader di quel Partito socialdemocratico che appoggia il governo Fanfani in carica dal 2I febbraio 1962. A questo punto, anche dopo le dichiarazioni di Palmiro Togliatti pronunciate una volta eletto Segni, risulta evidente per «L'Humanité» che compito del Pci è quello di creare un'unione con tutte le forze democratiche appartenenti alla sinistra ma anche a quella parte della Dc di sinistra che non ha accettato di mescolare i propri voti con quelli dei liberali, dei monarchici e dei missini per mandare al Quirinale Segni ${ }^{33}$.

Per «Forces Nouvelles» l'elezione di Segni è stata allo stesso modo chiarificatrice, ma ha delineato il quadro da un punto di vista diametralmente opposto. Infatti Moro, rifiutando la "proposta indecente» della coppia Togliatti-Nenni (quella cioè di votare Saragat per il Quirinale) si è opposto ad una "deriva» modello Fronte Popolare, che tendeva a «snaturare» il reale significato della formula di centro-sinistra. Il fallimento dell'operazione «Saragat Presidente»

[...] a permis de nettement délimiter les limites de la formule de centre-gauche appliquée par le gouvernement Fanfani, qui vise à réintégrer les socialistes, mais à isoler les communistes. En ne cédant pas devant le chantage de l'extrême-gauche, Aldo Moro a clairement démontré que les décisions du Congrès démo-chrétien de Naples n'étaient pas des clauses de style: oui à une alliance avec Nenni, non à la moindre coopération avec Togliatti ${ }^{34}$.

Ma «Forces Nouvelles» va oltre nella sua analisi e afferma che l'elezione di Segni costituisce un duro colpo anche all'ala «sinistra» della Dc, quella che in teoria vorrebbe una declinazione più «a sinistra» della formula di centro-sinistra e che avrebbe voluto vedere il fallimento dell'operazione Segni e non a caso ha rischiato di «logorarlo», non avendo garantito il suo voto ai primi scrutini. Anche le conclusioni di «Forces Nouvelles» sono interessanti, soprattutto ad uso del pubblico francese ed in particolare dell'elettorato e dei militanti Mrp, i quali stanno vivendo una difficile fase di tentativo di adattamento alle nuove istituzioni della Quinta Repubblica

33. A. Jacoviello, Pour la première fois depuis l'avènement de la République le chef de l'État a été élu grâce aux voix monarchistes et fascistes, "L'Humanité», 08/05/1962. Da notare che Jacoviello era in questa fase marito di Maria Antonietta Macciocchi, giornalista e poi deputata del Pci, piuttosto avulsa dalla linea ufficiale del partito. 34. P. Farine, L'élection difficile, «Forces Nouvelles», Io/05/1962. 
e alle nuove dinamiche politico-istituzionali successive all'avvicendamento a Matignon tra Debré e Pompidou.

Ajoutons à cela que la DC est un vaste mouvement regroupant de nombreuses tendances (en France on pourrait dire, en une grossière comparaison qu'un tel rassemblement irait de "La France catholique" à "Témoignage chrétien" et un peu au-delà des deux côtés) et que l'"ouverture à gauche" bouleverse profondément toutes les données classiques de la politique italienne ${ }^{35}$.

A «La Croix» sono meno assertivi nei giudizi, ma altrettanto convinti che l'elezione di Segni contribuisca a chiarire il significato del centrosinistra. Dopo aver affermato che proprio l'incertezza a proposito del nuovo esperimento italiano ha contribuito ad un'elezione presidenziale così laboriosa e travagliata, il quotidiano degli Assunzionisti conclude che se da un punto di vista generale a poco più di due mesi dal varo del nuovo governo Fanfani, è complicato comprendere fino a dove arriverà 'l'apertura a sinistra' delineata da Moro al Congresso Dc di Napoli, l'elezione di Segni costituisce un saldo argine affinché l'Italia non finisca per adottare politiche 'socialiste' ispirate da Nenni, se non addirittura da Togliatti ${ }^{36}$.

In questo quadro infine spicca poi il giudizio del quotidiano gollista «La Nation». L'organo dell'Unr si occupa solitamente poco di politica estera. Al contrario l'elezione di Segni alla presidenza della Repubblica è caratterizzata da quattro contributi tra il 2 e l'8 maggio. Le conclusioni sono molto meno assertive. "La Nation» non si sofferma più di tanto sulla figura di Segni, né descrive con particolari dettagli la lotta interna alle forze della maggioranza del governo Fanfani. Al contrario sottolinea più volte che il nuovo inquilino del Quirinale vi alloggia grazie ai voti dell'estrema destra fascista. Questo dovrebbe far riflettere sia sul futuro dell'esperienza di centro-sinistra, sia sui rischi di tenuta del sistema politico-istituzionale italiano $^{37}$.

\section{Il voto per la definitiva 'apertura'?}

Attese in Francia, ma non solo, per chiarire la reale dimensione politica della formula dell'apertura a sinistra, le elezioni del 28 aprile 1963 hanno un esito ben lontano da quello atteso e che apre una lunga fase di

35. Ibid.

36. G. Huber, M. Segni succède à M. Gronchi à la présidence de la République italienne. Une victoire de la volonté. Un échec pour M. Nenni, «La Croix», 08/05/1962.

37. Ouverture à gauche compromise?, «La Nation», 08/05/1962. 
instabilità politica e governativa, destinata a concludersi solo nel dicembre dello stesso anno ${ }^{38}$.

Ad emergere sono in particolare il pessimo risultato della Dc, per la prima volta abbondantemente sotto il $40 \%$, l'ottimo risultato del Pli (raddoppio dei voti come vero oppositore 'democratico' all'apertura a sinistra) e l'avanzata di quasi tre punti percentuali del Pci (con contestuale stagnazione del voto socialista). L'apertura a sinistra dunque bocciata dalle urne? Paradossalmente no, più o meno per tutti gli osservatori politici d'oltralpe ${ }^{39}$.

Nel senso che «L'Humanité» si concentra soprattutto sulla «vittoria» del Pci e in generale sulla constatazione che le forze di 'sinistra' nel loro complesso sono oramai maggioritarie nel Paese (senza soffermarsi sul carattere irrealizzabile di una maggioranza da Togliatti alla sinistra Dc passando per Saragat, da sempre strenuo anti-comunista). Proprio l'organo ufficiale del Pcf implicitamente accredita ciò che le altre forze politiche affermeranno in maniera esplicita: nonostante la sconfitta, la formula di centro-sinistra pare l'unica percorribile. Per certi versi «L'Humanité» la auspica, trattandosi proprio alla luce della recente consultazione elettorale, di una coalizione «debole e destinata ad andare rapidamente a pezzi» ${ }^{40}$.

Il gollista «La Nation» opta per un approccio più esplicito: nonostante il pessimo risultato dei partiti che la dovrebbero costituire (Psi compreso) l'apertura a sinistra è condannata ad andare avanti. Seppur debole è l'unica alternativa ad una pericolosa instabilità governativa. Centro-sinistra per forza, ma quale centro-sinistra? Bisogna però intendersi sulla definizione di apertura a sinistra o comunque di centro-sinistra. Secondo la pubblicazione gollista è indispensabile ripartire dal modello rappresentato dalla coalizione che ha sostenuto per circa un anno il governo Fanfani. Anche perché l'esito elettorale ha reso il rapporto con i socialisti nenniani alquanto complicato. Da un lato Nenni è portato a chiedere molto, soprattutto sui temi economico-sociali, in cambio del suo sostegno per non essere 'scavalcato' a sinistra. Dall'altro la Dc può concedere davvero poco a sinistra,

38. Con estrema attenzione «Le Monde» in una serie di quattro lunghi reportage del suo corrispondente dall'Italia Jean d'Hospital, pubblicati tra il I8 e il 29 aprile 1963, punta soprattutto l'attenzione sul mondo cattolico nel suo complesso, impegnato dal mese di ottobre del 1962 nello storico evento del Concilio Vaticano II e sulle sempre maggiori difficoltà da parte della Dc nel rappresentare l'interezza di questo composito mondo. La conclusione, nell'ultimo contributo, ricalca le considerazioni successive al Congresso Dc di Napoli del 1962: ancora una volta l'avvenire del Paese dipende dall'evoluzione del suo partito di maggioranza relativa.

39. Fuori dal coro l'analisi de «L'Express", che attribuisce al Psi di Nenni la vera responsabilità di veicolare la crisi successiva al voto di fine aprile 1963. "La crise du régime italien est ouverte, le PSI tient la clé de l'avenir. Une seule chose est certaine : cet avenir est à gauche» (M. Bosquet, Le rouge est mis, "L'Express", 09/05/1963). 40. A. Jacoviello, Crise au sein de la Démocratie chrétienne, "L'Humanité», Io/05/1963. 
dato che il successo dei liberali è un chiaro segnale lanciato dal suo elettorato più moderato e conservatore. Di grande interesse è anche la dimensione 'internazionale' del voto di fine aprile secondo "La Nation». Infatti l'insuccesso elettorale delle forze di governo può avere importanti ricadute sulla politica internazionale del Paese. La grande frammentazione politica contribuisce a fornire dell'Italia un'immagine debole. D'altra parte la proiezione di politica estera del Paese verrebbe altamente indebolita qualora un «internazionalista pacifista» come Nenni (peraltro anche anti-europeo) dovesse essere integrato nell'area di governo ${ }^{41}$.

Molto meno pessimista è «Forces Nouvelles», che sottolinea come la Dc debba affrontare l'esito elettorale con «sangue freddo» e ricordando che il risultato è comunque giunto seguendo la massima di De Gasperi a proposito del fatto che la «Dc è un partito di centro che guarda a sinistra». L'usura del potere è considerata un'altra delle cause che spiegano un risultato così modesto che però, unito anche all'avanzata del Pci, rende l'apertura a sinistra ancora più indispensabile. Serve però una «nuova apertura a sinistra», che non tema di richiamare il Psi di Nenni alle proprie responsabilità, soprattutto in relazione all'ambiguo rapporto con il Pci, abbandonato a livello nazionale, ma ancora saldo in molti contesti locali e a livello sindacale. Dal voto insomma devono emergere una Dc ancora più salda nelle sue convinzioni politiche e un Psi pronto ad una scelta di campo finalmente netta ${ }^{42}$.

\section{Il primo governo Moro e il centro-sinistra organico ${ }^{43}$ : un momento storico?}

Se vi è un elemento decisivo nei tentativi morotei di fornire un governo al Paese nel mese e mezzo successivo al voto di fine aprile, è certamente quello del cosiddetto 'politique d'abord'. Come correttamente ricorda ancora "Le Monde» a fine ottobre, su politica economica, politica estera e rapporto con il mondo comunista, le distanze tra democristiani e socialisti permangono e sono impossibili da riassorbire nello spazio di alcune settimane. A dominare è allora la dimensione "politica» e nello specifico "partitica». Moro su questo punto vince, piuttosto agevolmente, il braccio

4I. G. Broussine, L'Italie s'est condamnée à contrecœur à l'ouverture à gauche, «La Nation», 02/05/1963.

42. P. Farine, Un scrutin ambigu, «Forces Nouvelles», 09/05/1963.

43. Per avere un quadro esaustivo della complicata formazione del primo governo di centro-sinistra e dei suoi sei mesi di durata si rimanda al monumentale M. Franzinelli e A. Giacone, Il riformismo alla prova: il primo governo Moro nei documenti e nelle parole dei protagonisti, Milano, Feltrinelli, 2012. 
di ferro con Nenni ${ }^{44}$. Mentre la Dc non può permettersi una scissione, dal momento che è l'architrave del «sistema bloccato», sarà il Psi a dover fare un passo in più verso un'alleanza che, come giustamente ancora una volta ricorda «Le Monde», è oramai imposta da «motivazioni sistemiche» ${ }^{45}$. La Dc, seppur a rischio di frattura, non vuole tornare all'ipotesi di un centro-destra con liberali e socialdemocratici e allo stesso modo nessuno dei due partiti maggiori vuole tornare alle urne. Il paese necessita di un governo, possibilmente di legislatura (dopo la 'pausa' del gabinetto Leone) e dunque il centro-sinistra arriverà, non appena chiuso il congresso del Psi e certificata una scontata e imminente scissione in casa socialista ${ }^{46}$.

Al momento della presentazione al presidente Segni del governo di centro-sinistra finalmente 'organico', ancora il maggiore quotidiano francese parla di una «tappa storica, parte di un'evoluzione che non ha risparmiato nessuno dei grandi partiti italiani». Dopo aver elencato i «sostegni autorevoli» all'operazione, da Giovanni XXIII al nuovo pontefice Paolo VI passando per il compianto presidente statunitense J. F. Kennedy, «Le Monde» conclude che nell'accoglienza generale al nuovo governo e nell'entusiasmo di alcuni suoi ministri, si rivede quell' «ondata di fiducia» che aveva accolto i primi passi del governo di Pierre Mendès France nel I954. Un entusiasmo, quello di «Le Monde» che, soltanto una settimana dopo, sembra già scemare quando commentando l'intervento programmatico di Moro per la fiducia, si parla di una "prudente dichiarazione d'intenti» e ci si sofferma nuovamente a descrivere in dettaglio "la crisi interna a ciascuno dei due maggiori partiti della nuova coalizione di governo» ${ }^{47}$.

Anche la stampa di partito d'oltralpe segue con attenzione tutta la vicenda che dal tentativo di Moro del maggio 1963 conduce sino alla nascita del primo governo di centro-sinistra 'organico' del 4 dicembre 1963. Le posizioni e i giudizi si fanno sempre meno uniformi e per certi versi rispecchiano l'evoluzione dei soggetti politici francesi di riferimento. Seguendo allora l'asse destra-sinistra possiamo individuare ne «La Nation» il giudizio per certi versi più duro e attento a trovare nella nascita del governo Moro una svolta sistemica. Al momento della nascita del primo governo Dc-Psi-Psdi-Pri secondo il periodico gollista l'Italia si trova ad un bivio. L'estenuante rincorsa all'apertura a sinistra giunge a compimento nel mo-

44. Un interessante documento è il carteggio tra i due leader in questa delicata fase. Vedi P. Nenni, Aldo Moro carteggio 1960-1978, Firenze, La Nuova Italia, 1998.

45. Une crise en balance, "Le Monde», 31/10/1963.

46. M. Marchi, Centro-sinistra e storia nazionale, in G. Bernardini e M. Marchi (a cura di), A cinquanta anni dal primo centro-sinistra, cit., pp. I35-I45.

47. «Le Monde», I3/12/1963. 
mento in cui il cosiddetto 'miracolo economico italiano' vive una fase di appannamento. Il dominio assoluto della Dc nel periodo 1947-1963 ha permesso uno sviluppo e una ricostruzione pagati però, da un punto di vista politico, con la frammentazione, il parlamentarismo assoluto e la crescita costante delle sinistre, in particolare del Pci. Il bivio vero è a questo punto, conclude "La Nation», tra una svolta ancora più a sinistra (e una vera «socializzazione», sottointeso dei mezzi di produzione) e una cesura istituzionale verso una riforma in senso presidenziale, sottointeso sul modello di quella voluta da de Gaulle nel 1958 e completata sul finire del $1962^{48}$.

Il primo centro-sinistra organico è tutt'altro per «Forces Nouvelles». In questo caso siamo di fronte al trionfo della dimensione «europea» di quello che è definito una "grande svolta». Il successo dell' "ouverture à gauche» imporrebbe una seria riflessione al di là delle Alpi, anche perché fornisce l'opportunità all'Italia di sfuggire alla politica dei blocchi. Esplicitamente «Forces Nouvelles» afferma che una parte consistente dei dibattiti in corso in Francia (ci si riferisce alle ipotesi di avvicinamento tra Sfio-Mrp e mondo dei club in funzione anti-gollista) potrebbe trovare un'importante eco nel successo dell'apertura a sinistra in Italia.

Il faut suivre de près le déroulement de l'expérience italienne. Ce qui est en cause c'est la possibilité pour deux courants essentiels de la vie démocratique : le démocrate chrétien et le socialiste, non seulement de cohabiter en se neutralisant mais de coopérer dans une action commune. En choisissant de se séparer des communistes pour s'associer à la Démocratie chrétienne M. Nenni, l'un des plus anciens et des plus vigoureux leaders $d u$ socialisme européen, a fait un choix qui répondait à celui qu'avait fait la DC en refusant l'ouverture à droite. Il s'agit maintenant pour les uns et pour les autres de démontrer dans des actes de gouvernement la justesse de ce choix. L'intérêt d'une telle démonstration n'est pas limité à l'Italie ${ }^{4}$.

Tocca invece a «L'Humanité» occuparsi di chi, senza dubbio, paga il prezzo politico più alto dell'apertura e cioè il Psi di Nenni. Il quotidiano comunista giudica in maniera sarcastica il cosiddetto 'storico incontro' che, in realtà, certifica la vittoria su tutti i fronti della Dc morotea. Non solo il partito democristiano si è garantito i dicasteri più rilevanti nel primo governo guidato da Aldo Moro, ma quest'ultimo nel suo discorso di investitura, ha ribadito le pregiudiziali anticomunista e filo-atlantica del nuovo esecutivo, un tributo pagato ai settori più conservatori della Dc, parole pronunciate senza tenere in alcun conto le reazioni interne al Psi

48. P. Gilles, L'Italie à un carrefour. L'ouverture à gauche, «La Nation», 20/II/1963.

49. P. Farine, Le grand tournant, "Forces Nouvelles», 26/12/1963. 
nenniano. Ma è proprio su questo punto, conclude «L'Humanité», che il politico cattolico ha ottenuto la vittoria più schiacciante. $\grave{E}$ infatti riuscito ad indebolire il Psi, spingendolo ad una quasi certa scissione. Responsabile ultimo di questa situazione è Nenni, il quale ha scelto di puntare all'ingresso al governo, non preoccupandosi delle conseguenze nefaste per l'unità della sinistra in generale e del suo partito in particolare ${ }^{50}$.

La chiusura ancora una volta è per il quotidiano degli Assunzionisti. «La Croix» conferma la sua visione realista e quasi disincantata degli eventi che conducono alla nascita del primo governo Moro di centro-sinistra. Nei numerosi commenti nel corso della prima parte del mese di dicembre si descrive la fine estenuante di una lunga crisi che risale a prima del voto dell'aprile i963. E l'esito non può che essere una formula, sicuramente accattivante, sull'efficacia della quale si nutrono però non pochi dubbi. Secondo "La Croix», infatti, ogni singolo progetto del nuovo esecutivo sorretto dal Psi e con all'interno ministri socialisti, a cominciare dal leader Nenni, è una potenziale fonte di conflitto. In definitiva la governabilità è stata assicurata, perlomeno formalmente. La dimensione programmatica e l'efficacia 'riformatrice', sono legate ad un numero consistente di incognite, mediazioni e aggiustamenti, da effettuare sin dai primi passi del nuovo esecutivo, ma che potrebbero finire per svuotare di senso il significato stesso dell'intera operazione ${ }^{5 I}$.

\section{Considerazioni conclusive}

Francia e Italia dunque, nel momento in cui il resto dell'occidente euroatlantico entra a passi decisivi nella cosiddetta affuent society, si trovano ad affrontare due lunghe e complicate crisi sistemiche, che nel caso francese si conclude con un evidente cambio di regime, mentre nel caso italiano termina con una svolta 'solo' politica, con effetti sul funzionamento del complessivo sistema politico-istituzionale, ma senza alcuna nuova formalizzazione giuridico-costituzionale.

Nel complesso la stampa francese offre un'analisi attenta e puntuale della cosiddetta 'apertura a sinistra'. In particolare sono tre i punti più evidenziati rispetto alla svolta italiana. Da un lato la sua natura 'sistemica’, nonostante non si passi per la classica riforma della Costituzione.

50. A. Jacoviello, Les démocrates-chrétiens s'octroient tous les postes-clés du gouvernement cautionné par Nenni, "L'Humanité», 8/12/1963.

51. Moro a obtenu la confiance des députés italiens, «La Croix», I9/I2/1963. 
In secondo luogo è sottolineata più volte la 'specificità' della Democrazia cristiana come vero e proprio 'partito della Nazione', architrave di un sistema che cambia solo in funzione dei mutamenti lenti e delle evoluzioni interne allo stesso partito di maggioranza relativa. Infine, soprattutto nell'ultima fase, la sempre più evidente insistenza sulla 'inevitabilità' del centro-sinistra, ne finisce per sottolineare le evidenti contraddizioni interne e di conseguenza la diminuzione del suo valore di esempio come possibile svolta programmatica e riformista al centro dell'Europa occidentale. In definitiva più diventa 'indispensabile' e più il centro-sinistra si tramuta in una questione tutta interna all'esperienza italiana. Se anche in una prima fase lo si è immaginato come un'opzione esportabile, di sicuro tale ipotesi sfuma ed insieme a questa anche quella di una sua improbabile emulazione in terra francese. 